\title{
Concurrent Traffic Queuing Game in Smart Home
}

\author{
$1^{\text {st }}$ Maroua Ben Attia \\ University of Quebec \\ Quebec, Canada, H3C 1K3 \\ maroua.ben-attia.1@ens.etsmtl.ca
}

\author{
$2^{\text {nd }}$ Kim-Khoa Nguyen \\ University of Quebec \\ Quebec, Canada, H3C 1K3 \\ Kim-Khoa.Nguyen@etsmtl.ca
}

\author{
$3^{\text {rd }}$ Mohamed Cheriet \\ University of Quebec \\ Quebec, Canada, H3C 1K3 \\ mohamed.cheriet@etsmtl.ca
}

\begin{abstract}
Smart home gateway has to process different types of network traffic generated from several devices in an optimal way to meet their QoS requirements. However, the fluctuation of network traffic distributions results in packets concurrency. Current QoS-aware scheduling methods in the smart home networks do not consider concurrent traffic in their scheduling solutions. This paper presents an analytic model for a QoS-aware scheduling optimization of concurrent smart home network traffic with mixed arrival distributions and using probabilistic queuing disciplines. We formulate a hybrid QoS-aware scheduling problem for concurrent traffics in smart home network, and propose an innovative queuing design based on the auction economic model of game theory to provide a fair multiple access over different communication channels/ports. Our experiments show the proposed solution achieves an improvement of $14 \%$ of packets that meet their required delay and $57 \%$ of delay for different number of concurrent flows in the system.
\end{abstract}

Index Terms-Concurrent traffic, quality of service, smart home, traffic scheduling optimization

\section{INTRODUCTION}

As IoT (Internet of Things) and smart home applications flourish, the request for high-effective home networks with no congestion, less packet loss, and faster delay is significantly growing [1]. Smart home network connects different devices reacting with each other through heterogeneous wired and wireless physical accesses [2]. This class of devices includes sensors, home appliances, and multimedia devices and provides the home user with a large number of applications/services with different requirements in quality of service (QoS). Critical and delay-sensitive traffics, like medical, firedetector and video streaming traffics, should be processed first, however medium and low-priority traffics, like network management and best-effort traffics, may wait in the queue for a while before being processed by the home gateway [3]. Prioritizing high-priority traffics may lead to network congestion when all the same QoS-level traffics access to the gateway service simultaneously and with insufficient network bandwidth which can create several consistency problems like extra long delay or even packet loss. A large number of smart home devices generate periodically (like sensors), randomly (like detectors which work with triggering) and continuous data (like streaming devices) through the network making smart home networks more likely to experience concurrency issues. The dynamic nature of today's home network caused by the fluctuation of network traffic distribution raises the problem of flow concurrency where multiple devices send simultaneously their data to the home gateway, enlarging system payload, dropping flows and increasing scheduling latency.

Recent advances in optical, wireless and cellular modulation technologies have been made from the perspective of increasing the number of concurrents users per media access [4]. CSMA (carrier sense multiple access) widely used in existing systems, become less effective in delay for multi-channel/port concurrency issue. In addition, protocol design for multiChannel Concurrency techniques [5] does not consider the fairness between traffic flows from the same class of service which makes it unsuitable for delay-sensitive applications. Designing an efficient fair scheduling solution for concurrent packets belonging to different ethernet ports or different channels remains a challenging task. Thus, scheduling with QoS in the context of the smart home network should consider flow concurrency for both different and same media access and fair scheduling between same QoS-level concurrent traffic to avoid packet loss, local network congestion and ensure fair queuing between network flows. This requires automated management of traffic loads within the home gateway by offering multiple concurrent access for different channels/ethernet ports.

In this paper, we propose an analytic model for optimizing concurrent packet scheduling in a smart home network with mixed arrival distributions and different QoS requirements. We also contribute an innovative probabilistic queuing model for smart home networks which provides a fair scheduling between concurrent traffic belonging to different media access. The concurrent traffic schedule problem will then be modeled using an auction economic model of game theory and the solution is implemented on both traffic sources and the home gateway. The motivation behind the specific scheduling mechanism proposed for smart homes in this paper is two-fold: i) the specific traffic distribution in smart homes can be classified into three categories, as presented in Section III, while in the general context like the Internet it is normal distribution which is hard to model; and ii) the game theoretical model can be easily implemented in the home gateway serving a limited number of flows in the home. The rest of the paper is organized as follows. We will discuss related studies on QoS based scheduling in Section II. In Section III, we will describe the smart home traffic scheduling with concurrent flows. QoS scheduling problem is presented in Section IV. Section $\mathrm{V}$ describes the proposed queuing model for single QoS-level concurrent traffic in the smart home network (QC$\mathrm{SH})$. Performance results of our solution are provided in 
Section VI. Finally, we draw conclusions and present future work.

\section{RELATED WORK}

Various scheduling strategies have been deployed in smart home context to improve energy efficiency [6], [7], reduce power consumption [8] and improve response time [9]. However, the multi-channel/port concurrency issue has not yet been considered in the smart home network. The problem of providing concurrent accesses to a shared resource has been considered in several research areas; telecommunications [10], vehicular networks [11], computer systems [12], [13], etc. Zhang et al. [11] proposed a broadcast protocol for vehicular ad hoc networks (VANETs) which enables candidate forwarders in different transmission segments to concurrently transmit message packets. The authors used an accurate time synchronization mechanism to precisely calculate the packet forwarding time for each transmitter to satisfy concurrent transmissions requirements of orthogonal frequency division multiplexing (OFDM) signals in terms of the maximum temporal displacement. Despite the good performance provided by this solution in terms of the total broadcast delay, the large number of the concurrently transmitted messages can cause packet loss. Many efforts have been done to improve spatial multitasking either through adding additional resources like multiple CPU cores or by maximizing thread-level parallelism [12].

Game theory has been used in different research areas [14], [15]. An evolutionary game-theoretic approach is considered in [16] to reduce the average waiting time for local data processing units (LDPUs) in wireless body area network (WBAN).

A non-cooperative stochastic game is considered in [17] to bypass malicious nodes in cognitive radio networks. Since a normal/malicious unlicensed user attempts to maximize/minimize the expected average of the cumulative link utility along its selected path, the authors calculated a Nash equilibrium to select the channel which maximizes this utility. A coalition game-theoretic approach is used in [14] to solve the cluster formation problem in Device-to-Device (D2D) communications in 5G cellular networks. The game is used by the LTE base station to let the user join or leave a cluster based on its energy efficiency. Another coalition game is considered in [15] to enable full-duplex concurrent scheduling in millimeter wave wireless backhaul network. The game is used to find concurrently scheduled flows set with the maximum sum rate which maximizes the number of flows which satisfy their QoS requirements. In general, prior work focus on parallel executions that require high-performance computing [11], [13] and advanced hardware or protocol designs [10] that require significant hardware or protocol modifications. However, a home gateway is a limited-resource system with limited bandwidth and computational capabilities compared to 5G and WSN networks [18]. This makes it difficult to deploy such complex, time and space-consuming approaches on a smart home gateway. Also, multiple access solutions provided by multiplexing systems [4] enable simultaneous transmissions over only a single communication channel. Multi-Channel Concurrency (CCM) [5] in wireless systems allows concurrent multiple access over different channels from a single radio interface through using static or dynamic schedulers to control the switching frequency and the time allocation for each channel. These implementations cannot handle multi-channel/port concurrency issue.

Our approach mitigates these limitations by providing fair multiple access over different communication channels/ports smart-home network.

\section{SYSTEM DESCRIPTION}

A smart home allows the home user to control or remotely manage a network of smart devices. These devices can be classified into three main classes according to the temporal distribution of their network data:

a) Periodic data: These data are generated periodically by sensors and are generally used for monitoring. The sensors detect and send at each period of time the states of monitored devices to a central server to create models for analysis ends. These sensing objects include; connected thermostats, network sensors, medical sensors, etc., and generate traffics with a constant, determined distribution.

b) Random data: These data are generated randomly by sensors and usually used for notifications. The sensors generate traffic by triggering some events to notify the user or the application server by the abnormal activity in order to prevent dangerous threats. For example, a glass break detector that can measure the window pressure could notify the homeowner via his phone when someone attempting to break in, or a fire detector that can predict fires based on other sensors (like smoke sensors and temperature sensors) could ask homeowners for evacuation. These sensing objects generate random time independent traffics and then based on the Poisson process distribution [19] with an exponential interarrival rate.

c) Continuous data: These data are generated continuously with a very high arrival rate during peak periods of use and lower arrival rate during the rest of the day or by surveillance cameras. These objects includes; tablets, smartphones, connected televisions, surveillance cameras, etc. Home gateway has wireless and wired interfaces by which traffic will be redirected and routed from home network to the cloud. Each network device may communicate its generated data through Wi-Fi interface or Ethernet ports of the home gateway.

These data will be classified according to their priorities (QoS level), scheduled according to their arrived time and QoS level, and then served by the service module. Given the wide range of services provided in the smart home network with a different requirement in QoS, the fluctuation of network traffic distributions and a large number of smart devices, each network channel or ethernet port may be parallelly shared by more than one active flow having the same QoS level. To deal with the network flow concurrency issue, we add a bidding 
module at the entry of the principal queue. This module uses an auction game which prioritizes packets according to their bid values as described in Section V.

\section{QOS-AWARE SCHEdULING PROBLEM}

Our problem is optimizing QoS scheduling for concurrent network flows generated from different communication channels/ports which have the same QoS-level and the same arrival time while respecting the maximum tolerated delay required by their QoS level (unlike the existing access control techniques [4] that consider flow concurrency for only the same media access). The solution can be implemented on traffic sources as well as on the home gateway.

\section{A. System Model}

Incoming traffic can follow different distributions depending on their data type as well as the type of their generation process. We have a single domestic gateway with $c$ servers. A server can process any packet with a size up to the Maximum Transmission Unit (MTU). We assume that all packets are MTU-sized packets [20], [21] and the service follows a deterministic distribution [22], [23] with a rate $\frac{1}{s}$. Each data source can generate different QoS-levels of network traffic at different time slots, and a maximum of $k$ packets can be processed at each service time using $c$ servers. Our system is modeled as $G / D / c$. The service can serve:

- Up to $c$ packets in $s$ time slots,

- Up to $\frac{c}{s}$ packets in one time slot,

- Up to $\frac{c * D_{\max }\left(P_{i}\right)}{s}$ packets during the maximum required delay $D_{\max }\left(P_{i}\right)$ of a packet $P_{i}$.

Thus, for each packet $P_{i}$ we define a maximum window size $w_{\max }^{P_{i}}$ as the maximum number of packets that can be processed by the system's service during its required delay $D_{\max }\left(P_{i}\right)$ as follows:

$$
w_{\max }^{P_{i}}=\frac{c * D_{\max }\left(P_{i}\right)}{s}
$$

\section{B. Modeling concurrent traffic in smart home network}

Concurrent traffic $T=\left\{P_{i}^{q}, P_{i^{\prime}}^{q} \mid i \neq i^{\prime}, i, i^{\prime} \in R^{+}\right\}$are flows that have the same QoS-level pair $q=\left(p, w_{\max }\right)$ and that each has at least one packet $P_{i j}^{q} \in P_{i}^{q}$ and $P_{i^{\prime} j^{\prime}}^{q} \in P_{i^{\prime}}^{q}$ with the same arrival time slot t. We define the concurrent packets as $C=\left\{P_{i j}^{q}(t), P_{i^{\prime} j^{\prime}}^{q}\left(t^{\prime}\right) \mid P_{i j}^{q} \in P_{i}^{q}, P_{i^{\prime} j^{\prime}}^{q} \in P_{i^{\prime}}^{q}, t=t^{\prime}\right\}$ and the concurrent packet decision function as $D(C)$. Thus, the system will order the sequence of concurrent packet according to $\mathrm{D}(\mathrm{C})$. We also define $U\left(P_{i j}^{(q, g)}\right)$ the decision utility function as the gain of resources required for sending a packet $P_{i j}^{q}$ based on $D(C)$ function that we will determine later. The decision utility function is calculated as follows.

$$
U\left(P_{i j}^{(q, g)}\right)=\sum_{q=1}^{|Q|} \sum_{c=1}^{|C|} D_{T}\left(P_{c}^{q, g}\right)-D_{T}^{*}\left(P_{c}^{q, g}\right)
$$

where $D_{T}\left(P_{c}^{q, g}\right)$ and $D_{T}^{*}\left(P_{c}^{q, g}\right)$ are respectively the delay of processing the packet $\left(P_{c}^{q}\right)$ before and after applying the $D(C)$ function and are defined as the sum of waiting time in the queue and service time.

The smart home network is a heterogeneous infrastructure made of multiple electronic and electrical network devices like sensors, detectors, and laptops. These data sources generate a wide range of traffic with different distributions and various QoS requirements. A key challenge of this problem is to find an optimized schedule of multi-sourced and concurrent packets with respect to their QoS requirements. Thus, to meet the delay constraint, the delay of a packet $P_{i j}^{(q, S)}$ must be lower than the delay budget $D_{\max }^{q}$ required by the class of service $q$ :

$$
D_{T}\left(P_{i j}^{(q, S)}\right) \leq D_{\max }^{q}
$$

The QoS-aware scheduling problem consists of finding an optimal way to schedule packets from multi-sourced and concurrent traffic while ensuring their maximum tolerated delay and maximizing the concurrent-packet utility function $U\left(P_{i j}^{(q, g)}\right)$. We formulate the QoS-aware scheduling problem by the following objective function:

$$
(D(C))^{*}=\left\{\begin{array}{c}
\arg _{\max }\left(U\left(P_{i j}^{(q, g)}\right)\right), P_{i j}^{(q, g)} \in P \\
D_{T}\left(P_{i j}^{(q, g)}\right) \leq D_{\text {max }}^{q}
\end{array}\right.
$$

\section{QC-SH: QUEUING MODEL FOR SINGLE QOS-LEVEL CONCURRENT TRAFFIC}

The QC-SH, the innovative mechanism proposed in this paper, is inspired by the concept of auction used in game theory. We applied a bidding mechanism on concurrent packets to fairly schedule them with respect to their maximum tolerated delay required by their QoS level. Each source can place a bid based on the number of packets it wants to process. This bid is re-calculated at each bidding round using the previous bid result, and the maximum tolerated delay required by the source QoS level.

\section{A. Game Description}

QC-SH is based on a multi-player bidding mechanism. The players of the game are the concurrent packets $C$. They play for the first place in the media access to be processed first. There are $|C|=n$ players with valuations $v_{1}, \ldots, v_{n}$. In each bidding round, each player $P_{i j}^{q} \in C$ places a bid $b_{i j}^{q}$. The player with the maximum bid wins and will be processed by the system as well as its following $\mathrm{k}$ packets from $P_{i}^{q} \in T$.

At each bidding round $r$, the system compares the bidding values of the concurents flows and sends $\operatorname{sent}_{i j} * w_{\max }^{q}=$ $\left(v_{i j}-b_{i j}\right) * w_{\max }^{q}$ packets from the winning flow $P_{i j}^{q}$ using its bid value $b_{i j}$ and its valuation $v_{i j}$ at each round $r_{i}$ as follows:

$\operatorname{sent}_{i j}\left(r_{i}\right)= \begin{cases}v_{i j}\left(r_{i-1}\right)-b_{i j}\left(r_{i}\right) & \text { if } P_{i j}^{q} \text { wins round } r_{i} \\ 0 & \text { if } P_{i j}^{q} \text { looses round } r_{i}\end{cases}$

For each concurrent flow $P_{i}^{q} \in T$, we set a maximum window size $w_{\max }^{q}$ (see Eq.1) as the maximum number of packets that can be processed by the system's service during its required delay $D_{\text {max }}^{q}$. The initial valuation of a player $P_{i j}^{q}$ corresponds 
to $v_{i j}\left(r_{0}\right)=\frac{w_{\max }^{q}}{w_{\max }^{q}}=1$ and it is updated at each playing round as follows:

$v_{i j}\left(r_{i}\right)=\left\{\begin{array}{cc}v_{i j}\left(r_{i-1}\right)-\operatorname{sent}_{i j}(r) & \text { if } P_{i j}^{q} \text { wins round } r_{i} \\ v_{i j}\left(r_{i-1}\right)+\operatorname{sent}_{i j}(r) & \text { if } P_{i j}^{q} \text { looses round } r_{i}\end{array}\right.$

If a packet $P_{i j}^{q}$ wins a round, its valuation $v_{i j}$ decreases by the number of sent packets by the system, but if it loses a round, its valuation increases to cover its loss from the previous round and to increase its winning probability. Thus, the probability of successful processing of packets of a source increases with its previous loss rate. For each concurrent packet $P_{i j}^{q} \in C$, we define a window size $w_{i j}^{q}$ as the number of sub packets $P_{i j}^{q}$ to be processed by the system during the delay of $P_{i j}^{(q, g)}$ as follows:

$$
w_{i j}^{q}=\frac{c * D\left(P_{i j}^{(q, g)}\right)}{s}
$$

Whether a player wins or looses, the system calculates its

utility $U_{i j}^{q}$ at each playing round $r_{i}$ as follow:
$U_{i j}^{q}\left(r_{i}\right)=P r_{w i n}^{i j}\left(r_{i}\right) * \frac{\operatorname{sent}_{i j}\left(r_{i}\right)}{w_{\max }^{q}}=P r_{w i n}^{i j}\left(r_{i}\right) *\left[v_{i j}\left(r_{i-1}\right)-b_{i j}\left(r_{i}\right)\right]_{\leftrightarrow} \rightarrow(n-1)[F(v)]^{n-2} f(v)[v-b(v)]-b(v)^{\prime}[F(v)]^{n-1}=0$

where $P r_{\text {win }}^{i j}\left(r_{i}\right)$ is the winning probability of packet $P_{i j}^{q}$ in round $r_{i}$

\section{B. Game Model}

As described before, the system sends $v_{i j}-b_{i j}$ packets from the winner flow. Thus, the system wants to maximize the bid value to achieve some fairness between concurrents traffic by sending a few packets from each flow to increase the probability of winning for other players. However, concurrent flows want to minimize their bid to send a maximum number of packets from its flow. Based on the tradeoff between the selected winner and the number of processed packets, we model our game as follows:

- Type: First price auction game. It allows the concurrent traffic to choose its strategy based on its window size.

- Players: A finite set $N$ of concurrent traffics from different media access.

- The resource that the palyers play for: The media access to transmit its packets over the acquired priorities.

- Game coordinator: The home gateway

- Strategies: $S=$ set of actions $=R^{+}$

- Initial valuation: $v_{i j}\left(r_{0}\right)=\frac{w_{\max }^{q}}{w_{\max }^{q}}=1$

- Winner: the player with the highest bid

- Utility: $U_{i j}(v, b)=1\left(b_{i} \geq \max _{j \neq i} b_{j}\right)\left(v_{i j}-b_{i j}\right)$

- Duration of the game: $T=\varepsilon+k \delta$; with $k$ is the total number of packets to be sent concurrently, $\delta$ is the duration of one packet (=MTU), $\varepsilon$ is game computation, evaluation, and analyses times.

\section{Nash Equilibrium}

The valuations $v_{i}$ of the concurrent flows are independent and identically distributed across the players and follow the uniform distribution on $[0,1] . b^{*}(v)$ is a symmetric Bayesian nash equilibrium (BNE) for each concurrent packet $P_{i j}^{q}$ if $b^{*}\left(v_{i j}\right)$ is its best response when all other players bid $b^{*}\left(v_{i^{\prime} j^{\prime}}\right)$. The existence of a BNE in First price auction is proven by Lebrunen in [24]. Let $F$ and $f$ be respectively the general cumulative distribution function (strictly increasing) and the probability density function. We assume that the symmetric BNE $b *$ is strictly increasing and differentiable. The utility of the packet $P_{i j}^{q}$ with valuation $v_{i j} \in[0,1]$ and bid $b(w) \in[0,1]$ is as follows:

$$
\begin{aligned}
U & =\operatorname{Pr} r_{w i n}[v-b(w)]=\operatorname{Pr}\left(\max _{j \neq i} b(j) \leq b(w)\right)[v-b(w)] \\
& =[F(w)]^{n-1}[v-b(w)]
\end{aligned}
$$

$$
\frac{\partial U}{\partial w}=(n-1)[F(w)]^{n-2} f(w)[v-b(w)]-b(w)^{\prime}[F(w)]^{n-1}
$$

The utility is maximized using the first order condition described in Eq.11:

$$
\begin{aligned}
& \left.\frac{\partial U}{\partial w}\right|_{\mid w=v}=0 \\
& \leftrightarrow(n-1)[F(v)]^{n-2} f(v)[v-b(v)]-b(v)^{\prime}[F(v)]^{n-1}=0
\end{aligned}
$$

Thus, $b^{*}(v)=\frac{v(n-1)}{n}$ is a symmetric Bayesian nash equilibrium for the game since it maximizes the utility given any valuation $v$.

$$
\begin{array}{rl}
\int_{0}^{x} \frac{\partial U}{\partial w} \mid w=v & \mathrm{~d} v=0 \\
\leftrightarrow & {\left[F(v)^{n-1} * v\right]_{0}^{x}-\int_{0}^{x} F(v)^{n-1} \mathrm{~d} v-\left[F(v)^{n-1} * b^{*}(v)\right]_{0}^{x}=0} \\
\leftrightarrow & v * F(v)^{n-1}-\frac{v^{n}}{n}-b^{*}(v) * F(v)^{n-1}=0 \\
\leftrightarrow & v^{n}-\frac{v^{n}}{n}-b^{*}(v) * v^{n-1}=0 \\
\leftrightarrow & b^{*}(v)=\frac{v^{n}-\frac{v^{n}}{n}}{v^{n-1}} \leftrightarrow \quad \leftrightarrow \quad b^{*}(v)=\frac{v(n-1)}{n}
\end{array}
$$

The auction game model proposed under concurrency issue is called QC-SH.

\section{PERformance Evaluation}

\section{A. Experimental Setup}

To evaluate the performance of the proposed QC-SH algorithm, the experiment was carried out in a simulated network environment similar to the smart home network one. We build a simulation with up to 200 concurrent flows, each has flow $_{\text {size }}=20$ network packets (a total of 4000 packets). The flows are processed using 4 servers and with a service time $=5 \mathrm{~ms}$. The maximum delay required by the service class $q$ of concurrent flows is $D_{\max }^{q}=40 \mathrm{~ms}$. We calculate the maximum number of packets $w_{\max }$ that can be processed by 

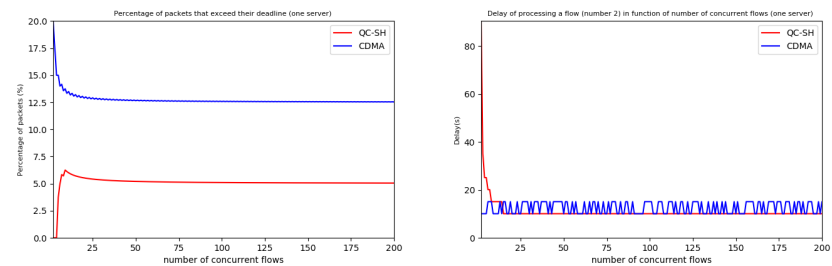

(a) Percentage of packets that exceed (b) Delay of processing flow number their deadline in function of number 2 in function of number of concurof concurrent flows (\%) rent flows (second)

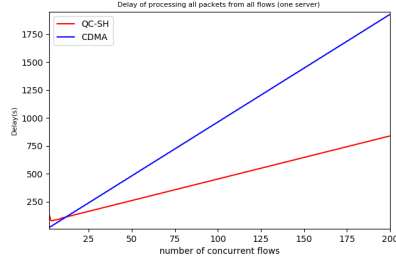

(c) Delay of processing each flow in function of number of concurrent flows (second)

Fig. 1. QC-SH performances in function of number of concurrent flows (one server)
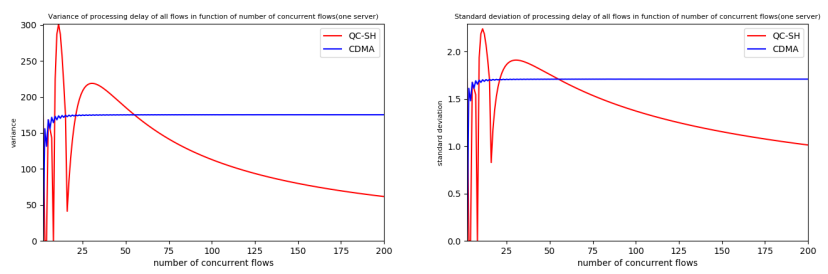

(a) Variance of processing delay of all flows in function of number of concurrent flows (second)

(b) standard deviation of processing delay of all flows in function of number of concurrent flows (second)

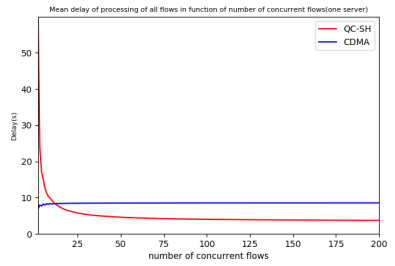

(c) Mean delay of processing of all flows in function of number of concurrent flows (second)

Fig. 2. Statistical performances for QC-SH in function of number of concurrent flows (one server)

the system's service during the required delay $D_{\max }$ as defined in Eq.(1).

We test our QC-SH algorithm with one and four servers and we compare it with recent mono-processing and multiprocessing scheduling approaches as follow:

- Mono-processing approaches: Theses approaches con-
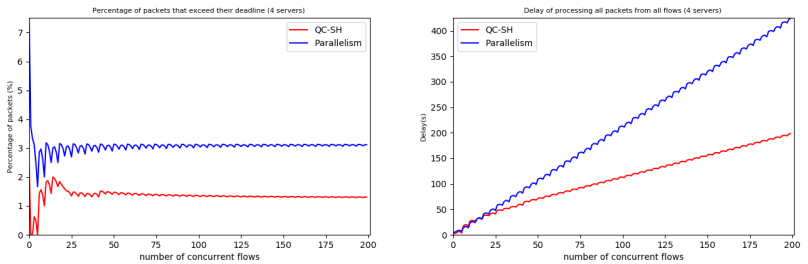

(a) Percentage of packets that exceed their deadline in function of

(b) Delay of processing all packets number of concurrent flows $(\%)$ rom all flows in function of number of concurrent flows (second)
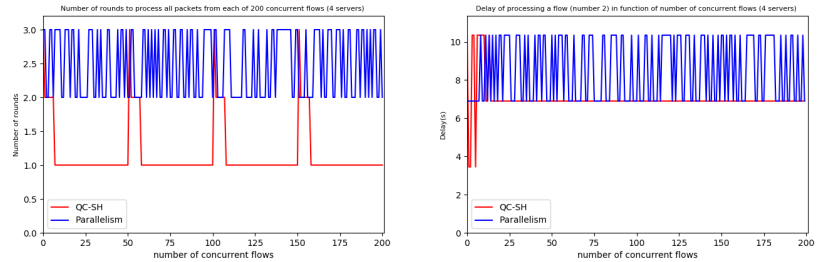

(c) Number of rounds to process all pakets from each of 200 concurrent flows

(d) Delay of processing flow number 2 in function of number of concurrent flows (second)

Fig. 3. QC-SH performances in function of number of concurrent flows (4 servers)

sider a single server. They include TDMA solution [15](it serially transmits packets) and concurrency-based scheduling solutions like CDMA [4], STDMA [15], SFD [15], and others [4], [11], [15]. In our experimentation, we use $C D M A$ approach as a candidate to refer to this group since all channels/ports concurrent approaches are based on the CDMA solution. CDMA allows multiple access over a single media access (channel/port). However, for concurrent traffic over multi-channels/ports CDMA performs as TDMA as it processes a single packet in each media access. With a focus on multi-channels/ports concurrency issue, we simulate the CDMA approach by serially transmit concurrent packets for each channel/port. We setup the CDMA approach using 1 server and 200 concurrent flows.

- Multiprocessing approaches: These approaches consider more than one server. They include concurrency-based scheduling solutions that allow spatial multitasking using multiple CPUs [18]. We refer to this group Parallelism and we setup it using 4 servers and 200 concurrent flows.

\section{B. Experimental Results}

Fig. 1 shows the performances of the proposed QC-SH algorithm in terms of the number of concurrent flows compared to the CDMA approach [4] using a single server. Three metrics are considered: a) the percentage of packets that exceed their deadline (Fig. 1(a)), b) the delay of processing a single flow (we test with the flow number 2 as it is always present for different number of concurrents flows; from 2 to 200) (Fig. 1(b)), and c) the delay of processing each flow (Fig. 1(c)). We can see our solution provides lower delay 

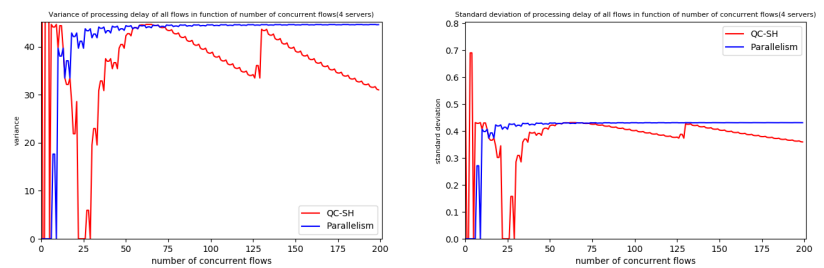

(a) Variance of processing delay of (b) standard deviation of processing all flows in function of number of delay of all flows in function of concurrent flows (second) number of concurrent flows (second)

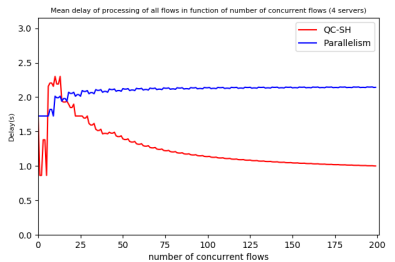

(c) Mean delay of processing of all flows in function of number of concurrent flows (second)

Fig. 4. Statistical performances for QC-SH in function of number of concurrent flows (4 servers)

for processing all flows and a lower number of packets that exceed their deadline compared to CDMA. QC-SH provides a minimum of $94 \%$ of packets that respect their maximum delay compared to $80 \%$ with CDMA (an improvement of $14 \%$ ). The processing delay of all flows with QC-SH is lower than that with CDMA (Fig. 1(c)). This can be explained by the fairness feature of our solution in order to provide a lower total processing delay for concurrent flows. As shown, QC-SH processes up to 200 concurrent flows in a maximum of $750 \mathrm{~s}$, with around 10s and 2 processing rounds per packet) compared to up to 1750 s with CDMA, with around 18 s and 4 processing rounds per packet (an improvement of 14\%). Therefore, our approach performs better in heavy concurrent traffic condition which is the case of smart home network. We also study the fairness of processing concurrent flows using the statistical parameters of the proposed QC-SH in terms of the number of concurrent flows and using a server (Fig. 2). We compare the statistical performances of the proposed QC-SH to the CDMA solution in terms of a) the variance (Fig. 2(a)), b) the standard deviation (Fig. 2(b)), and c) the mean delay of processing of all flows (Fig. 2(c)). When we increase the number of concurrent flows, both the variance and the standard deviation of the processing delay with QC-SH decrease while remaining under CDMA curves. When we increase the number of concurrent flows, the processing delay of all flows will be close to the average. We conclude that the fairness feature of our solution makes it less sensitive to the fluctuation of the number of concurrent traffics. Furthermore, QC-SH experiences a lower mean delay for any number of concurrent flows compared to the CDMA solution. We note that at the beginning of the experiment (with the lower number of concurrent flows) QC$\mathrm{SH}$ has high variance, standard deviation and mean delay. This can be explained by the extra overhead resulting from game computation, evaluation, and analyzing times. Fig. 3 shows the performances of the proposed QC-SH algorithm in terms of the number of concurrent flows compared to the Parallelism approach using 4 servers. Four performance metrics are considered: a) the percentage of packets that exceed their deadline (Fig. 3(a)), b) the delay of processing flow number 2 (Fig. 3(b)), c) the number of rounds to process all pakets from each of 200 concurrent flows (Fig. 3(c)), and d) the delay of processing each flow (Fig. 3(d)). We observe the QC-SH works better with 4 servers (with an improvement of up to $4 \%$ in packets that respect their maximum delay, $600 \mathrm{~s}$ in delay of processing all concurrent flows, one processing round per packet and $3 \mathrm{~s}$ of processing delay per packet compared to QC-SH with one server). We also note that QC-SH performs better than the Parallelism solution [18] with an improvement of up to $2 \%$ in terms of the number of packets that preserve their maximum delay, up to $250 \mathrm{~s}$ in delay of processing all concurrent flows, two processing rounds per packet and $3.5 \mathrm{~s}$ of processing delay per packet. Therefore, our approach performs better with multiple servers. In Fig. 4, we study statistical performances of the proposed QC-SH in terms of the number of concurrent flows compared to the Parallelism solution [18] using four servers in terms of a) the variance (Fig. 4(a)), b) the standard deviation (Fig. 4(b)), and c) the mean delay of processing of all flows (Fig. 4(c)). We see an improvement of QC-SH with 4 servers of $83 \%$ in variance, $90 \%$ in standard deviation and $88 \%$ in mean delay compared to QC-SH with one server. In addition, QC-SH performs better than the Parallelism solution [18] in terms of the fairness of processing flows with lower variance, standard deviation and mean delay for different numbers of concurrent flows.

\section{CONCLUSION}

In this paper, we proposed a new probabilistic queuing model for concurrent smart home network traffic over different communication channels/ports, to provide some fairness in processing concurrent flows and increase the number of packets that meet their deadline while decreasing the total processing delay. We tested our solution with 4000 network packets and 200 concurrent flows using one and four servers. Then, we compared it to the recent mono-processing and multiprocessing based scheduling solutions for each criterion. Our experimental results demonstrated that the proposed algorithm outperforms the current solutions in almost the totality of criterion. Future work could include the design of an overheadfree model for our QC-SH mechanism using different packet sizes and different service distributions. We will further address the problem when flows provide untrue information to gain an advantage in the game (some applications may set a lower maximum delay than necessary in the game). Also, our current results showcase only our approach over simulating networking settings, which will also be extended to incorporate actual modern smart home networks with real network data, 
monitoring, and user applications, combined with real QoS requirement specifications.

\section{REFERENCES}

[1] Y. Qi, J. Wu, G. Gong, J. Fan, A. Orlandi, W. Yu, J. Ma, and J. L. Drewniak, "Notice of retraction: Review of the emc aspects of internet of things," IEEE Transactions on Electromagnetic Compatibility, vol. 60, no. 5, pp. 1152-1160, Oct 2018.

[2] W. M. Khan and I. A. Zualkernan, "Sensepods: A zigbee-based tangible smart home interface," IEEE Transactions on Consumer Electronics, vol. 64, no. 2, pp. 145-152, May 2018.

[3] D. Kotani, "An architecture of a network controller for qos management in home networks with lots of iotdevices and services," in 2019 16th IEEE Annual Consumer Communications Networking Conference (CCNC), Jan 2019, pp. 1-4.

[4] S. Han, Y. Liang, B. Soong, and S. Li, "Dynamic broadband spectrum refarming for ofdma cellular systems," IEEE Transactions on Wireless Communications, vol. 15, no. 9, pp. 6203-6214, Sep. 2016.

[5] K. Anand, S. J. Kuhn, and A. Raissinia, "Dynamic scheduling for multi-channel concurrency switching," Apr. 16 2015, uS Patent App. $14 / 172,824$.

[6] B. Zhou, W. Li, K. W. Chan, Y. Cao, Y. Kuang, X. Liu, and X. Wang, "Smart home energy management systems: Concept, configurations, and scheduling strategies," Renewable and Sustainable Energy Reviews, vol. 61, pp. 30 - 40, 2016. [Online]. Available: http://www.sciencedirect.com/science/article/pii/S1364032116002823

[7] X. Chen, T. Wei, and S. Hu, "Uncertainty-aware household appliance scheduling considering dynamic electricity pricing in smart home," IEEE Transactions on Smart Grid, vol. 4, no. 2, pp. 932-941, June 2013.

[8] S. Khan, Z. A. Khan, N. Javaid, S. M. Shuja, M. Abdullah, and A. Chand, "Energy efficient scheduling of smart home," in Web, Artificial Intelligence and Network Applications, L. Barolli, M. Takizawa, F. Xhafa, and T. Enokido, Eds. Cham: Springer International Publishing, 2019, pp. 67-79.

[9] J. Leu, C. Chen, and K. Hsu, "Improving heterogeneous soa-based iot message stability by shortest processing time scheduling," IEEE Transactions on Services Computing, vol. 7, no. 4, pp. 575-585, Oct 2014.

[10] S. Wang, S. M. Kim, L. Kong, and T. He, "Concurrent transmission aware routing in wireless networks," IEEE Transactions on Communications, vol. 66, no. 12, pp. 6275-6286, Dec 2018.

[11] X. M. Zhang, L. Yan, H. Zhang, and D. K. Sung, "A concurrent transmission based broadcast scheme for urban vanets," IEEE Transactions on Mobile Computing, vol. 18, no. 1, pp. 1-12, Jan 2019.

[12] J. Kim, J. Cha, J. J. K. Park, D. Jeon, and Y. Park, "Improving gpu multitasking efficiency using dynamic resource sharing," IEEE Computer Architecture Letters, vol. 18, no. 1, pp. 1-5, Jan 2019.

[13] Y. Wang, W. Chen, J. Yang, and T. Li, "Exploiting parallelism for cnn applications on $3 \mathrm{~d}$ stacked processing-in-memory architecture," IEEE Transactions on Parallel and Distributed Systems, vol. 30, no. 3, pp. 589-600, March 2019.

[14] A. Asadi and V. Mancuso, "Network-assisted outband d2d-clustering in $5 \mathrm{~g}$ cellular networks: theory and practice," IEEE Transactions on Mobile Computing, vol. 16, no. 8, pp. 2246-2259, 2017.

[15] H. Jiang, Y. Niu, J. Zhang, B. Ai, and Z. Zhong, "Coalition game based full-duplex concurrent scheduling in millimeter wave wireless backhaul network," China Communications, vol. 16, no. 2, pp. 59-75, Feb 2019.

[16] S. Misra and S. Sarkar, "Priority-based time-slot allocation in wireless body area networks during medical emergency situations: An evolutionary game-theoretic perspective," IEEE Journal of Biomedical and Health Informatics, vol. 19, no. 2, pp. 541-548, 2015.

[17] W. Wang, A. Kwasinski, D. Niyato, and Z. Han, "Learning for robust routing based on stochastic game in cognitive radio networks," IEEE Transactions on Communications, vol. 66, no. 6, pp. 2588-2602, 2018.

[18] W. Ding, Y. Niu, H. Wu, Y. Li, and Z. Zhong, "Qos-aware full-duplex concurrent scheduling for millimeter wave wireless backhaul networks," IEEE Access, vol. 6, pp. 25 313-25 322, 2018.

[19] E. Grigoreva, M. Laurer, M. Vilgelm, T. Gehrsitz, and W. Kellerer, "Coupled markovian arrival process for automotive machine type communication traffic modeling," in 2017 IEEE International Conference on Communications (ICC), May 2017, pp. 1-6.
[20] N. G. Nayak, F. Dürr, and K. Rothermel, "Time-sensitive softwaredefined network (tssdn) for real-time applications," in Proceedings of the 24th International Conference on Real-Time Networks and Systems, ser. RTNS '16. New York, NY, USA: ACM, 2016, pp. 193-202. [Online]. Available: http://doi.acm.org/10.1145/2997465.2997487

[21] P. Orosz, T. Skopkó, and M. Varga, "Rctp: A low complexity transport protocol for collecting measurement data," Infocommunications Journal, vol. 6, no. 3, pp. 28-36, 2014.

[22] X. Chen, Z. Li, Y. Chen, B. Du, and Y. Zhang, "Traffic modeling and performance evaluation of sdn-based nb-iot access network," Concurrency and Computation: Practice and Experience, p. e5145.

[23] F. Metzger, T. Hoßfeld, A. Bauer, S. Kounev, and P. E. Heegaard, "Modeling of aggregated iot traffic and its application to an iot cloud," Proceedings of the IEEE, vol. 107, no. 4, pp. 679-694, 2019.

[24] B. Lebrun, "Existence of an equilibrium in first price auctions," Economic Theory, vol. 7, no. 3, pp. 421-443, Oct 1996. [Online]. Available: https://doi.org/10.1007/BF01213659 